\title{
Die berufliche Vorsorge im Rahmen von M\&A Transaktionen - eine Übersicht
}

Unternehmensverkäufe und Restrukturierungen haben nicht nur direkte Folgen für Mitarbeitende, sondern auch für die Vorsorgeeinrichtungen, bei denen die Mitarbeitenden versichert sind. Unter bestimmten Voraussetzungen kann es zu einer teilweisen organisatorischen Aufhebung - einer Teilliquidation - der Vorsorgeeinrichtung kommen. Um Ungleichheiten zu vermeiden, sind Vorsorgeeinrichtungen gesetzlich verpflichtet, in einem Reglement die Voraussetzungen und das Verfahren einer solchen Teilliquidation zu regeln. Die betroffenen Mitarbeiter sind vor allem an der Höhe der Austrittsleistung interessiert und wollen am Erfolg und an den Reserven beteiligt werden. Die Vorsorgeeinrichtung verfolgt jedoch genau gegenläufige Interessen und will das Substrat soweit wie möglich behalten. Aus Sicht der Mitarbeiter gibt es darum bei Teilliquidationen von Vorsorgeeinrichtungen einige Besonderheiten zu beachten.

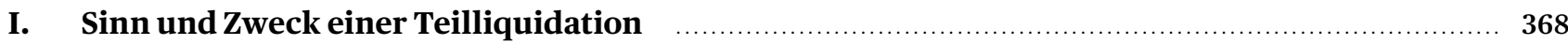

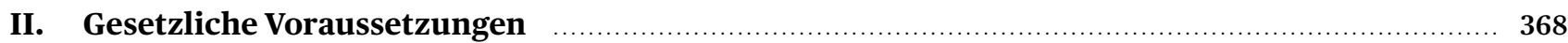

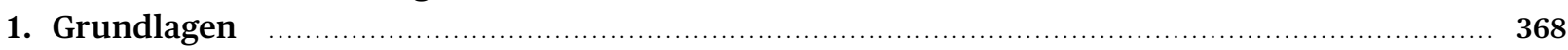

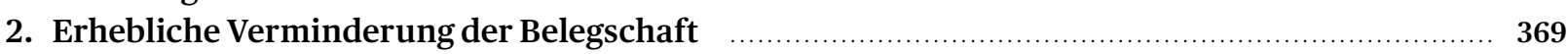

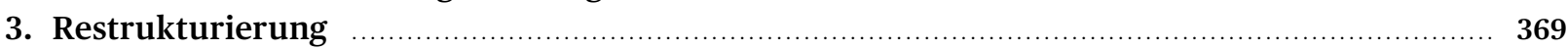

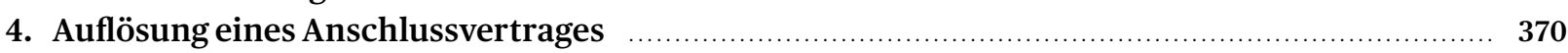

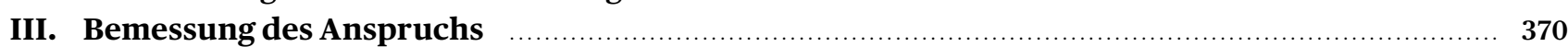

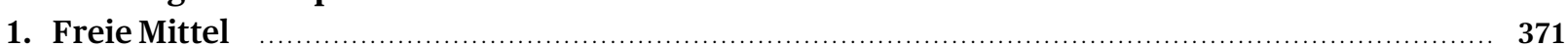

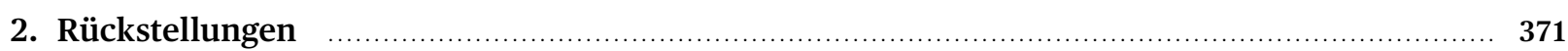

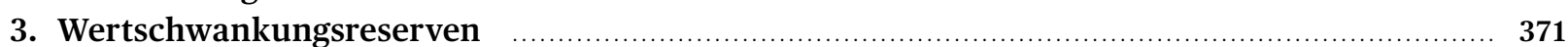

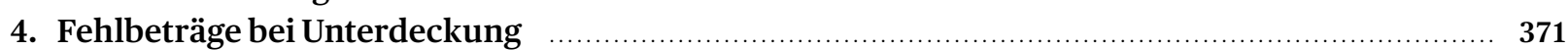

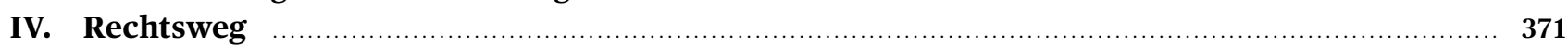

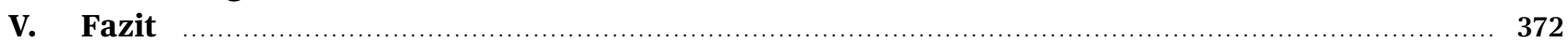

Zitiervorschlag:

MARC NUFER / LORENZ RAESS, Die berufliche Vorsorge im Rahmen von M\&A Transaktionen - eine Übersicht, sui generis 2020, S. 367

Marc Nufer, Rechtsanwalt, Partner, Head Corporate / M\&A (marc. nufer@eversheds-sutherland.ch); Lorenz Raess, Rechtsanwalt, Associate, Corporate / M\&A (lorenz.raess@eversheds-sutherland.ch); beide bei Eversheds Sutherland AG.

URL: sui-generis.ch/146

DOI: https://doi.org/10.21257/sg.146

Dieses Werk ist lizenziert unter einer Creative Commons Namensnennung - Weitergabe unter gleichen Bedingungen 4.0 International Lizenz. 


\section{Sinn und Zweck einer Teilliquidation}

1 Höhe und Umfang des angesparten Vorsorgevermögens sind ein Aspekt, der bei M\&A Transaktionen und Restrukturierungen häufig in den Hintergrund rückt. Das Bundesgericht hat wiederholt betont, dass dieses Vermögen den versicherten Mitgliedern (den Destinatären) zu folgen hat. ${ }^{1}$ Da die Destinatäre einer Vorsorgeeinrichtung (VE) es mit ihren Beiträgen überhaupt erst ermöglichen, dass Geld gewinnbringend angelegt werden kann, sollen sie deshalb nicht nur erhalten, was sie einbezahlt haben, sondern darüber hinaus auch anteilsmässig am Gewinn beteiligt werden. ${ }^{2}$ Ansonsten würden nur die verbleibenden Destinatäre davon profitieren. ${ }^{3}$ Je nach der Anzahl der austretenden Destinatäre und des betroffenen Vorsorgevermögens ist vorgesehen, dass eine VE teilliquidiert werden muss.

2 Vereinfacht gesagt will die Teilliquidation (TL) einer VE eine Über- und Unterkapitalisierung verhindern. Einerseits sollen verbleibende Destinatäre nicht auf Kosten der Austretenden profitieren. Andererseits sollen die verbleibenden Destinatäre nicht alleine die Fehlbeträge einer Unterdeckung tragen. ${ }^{4}$

3 Die Interessen der Destinatäre und der VE selber sind in diesem Fall jedoch gegensätzlich. In wirtschaftlich guten Zeiten lohnt es sich für die Destinatäre, im Rahmen einer TL kollektiv aus der VE auszuscheiden, damit sie am Erfolg partizipieren. Sind die Zeiten hingegen weniger rosig, ist ein individueller Ausritt ausserhalb einer TL vorteilhafter, da nicht mit einer Kürzung der Austrittsleistung gerechnet werden muss. ${ }^{5}$ Demgegenüber will die VE in erfolgreichen Zeiten möglichst keine TL durchführen und das angehäufte Kapital behalten. Bei einer Unterdeckung ist eine TL aber vorteilhafter, um Austrittsleistungen zu kürzen und möglichst viel Vor-

\footnotetext{
1 BGE133V607 E. 4.2.1.

2 BGE143V 200 E. 4.2.3; BGE128 II 397 E.3.2; SABINA WILSON, Die Erstellung des Teilliquidationsreglements einer Vorsorgeeinrichtung und weitere Einzelfragen zur Durchführung einer Teilliquidation, Diss. Basel 2016, Rz. 5.

3 Das Ganze gilt auch bei einer Unterdeckung, wo Fehlbeträge zu einem gewissen Grad abgezogen werden können (Art. 19 Abs. 2 Bundesgesetz über die Freizügigkeit in der beruflichen Alters-, Hinterlassenen- und Invalidenvorsorge vom 17. Dezember 1993 [Freizügigkeitsgesetz, FZG; SR 831.42]; Art. 53d Abs. 3 Bundesgesetz über die berufliche Alters-, Hinterlassenen- und Invalidenvorsorge vom 25.Juni 1982 [BVG; SR 831.40]; Art. 44 Verordnung über die berufliche Alters-, Hinterlassenen- und Invalidenvorsorge vom 18. April 1984 [BVV 2; SR 831.441.1]).

4 MONIKA STOCKER, Die Teilliquidation von Vorsorgeeinrichtungen: Unter besonderer Berücksichtigung der Härtefallproblematik bei Teilliquidationen in Unterdeckung, Diss. Fribourg 2012, S. 71.

5 STOCKER (Fn.4), s.70 f.
}

sorgekapital in der VE zu erhalten. Erfolgen bei einer Unterdeckung zu viele Austritte ausserhalb einer TL, kann das aufgrund der fehlenden Möglichkeiten der Kürzungen ansonsten zu erheblichen finanziellen Engpässen der VE führen. ${ }^{6}$

\section{Gesetzliche Voraussetzungen}

\section{Grundlagen}

Bei einem einfachen Freizügigkeitsfall - sofern kein Vor- 4 sorgefall eingetreten ist - hat der ausscheidende Destinatär nur Anspruch auf seine einbezahlten Mittel, nicht mehr und nicht weniger. Da ein einzelner Austritt im Normalfall nur zu einer geringen Veränderung des gesamten Vorsorgekapitals führt, rechtfertigt sich eine TL in diesem Fall aus Praktikabilitätsgründen nicht. ${ }^{7}$ Wie gleich gezeigt wird, kommt eine TL erst bei der Erfüllung gewisser quantitativer Merkmale zum Zug.

Gemäss dem Freizügigkeitsgesetz (FZG) besteht im Falle 5 einer TL neben dem Anspruch auf die Austrittsleistung8 auch ein solcher auf die freien Mittel der VE. ${ }^{9}$ Dieser Anspruch kann individueller oder kollektiver Natur sein. Der individuelle Austritt innerhalb einer TL erfolgt durch mehrere Personen, die jedoch im Anschluss nicht in die gleiche VE wechseln. Beim kollektiven Austritt wechselt eine ganze Gruppe in eine andere VE. In letzterem Fall haben die Destinatäre zudem per Gesetz das Recht, sich anteilsmässig an den Rückstellungen und Schwankungsreserven zu beteiligen. ${ }^{10} \mathrm{Ab}$ wann jedoch ein kollektiver Austritt vorliegt, ist gesetzlich nicht festgehalten und muss weiter konkretisiert werden.

Das Bundesgesetz über die berufliche Alters-, Hinterlas- 6 senen- und Invalidenvorsorge (BVG) sieht in Art. 53b Abs. 1 vor, dass die jeweiligen VE in einem Reglement die Voraussetzungen und das Verfahren einer TL regeln müssen. ${ }^{11}$ Das TL-Reglem ent ist von der jeweiligen kantonalen Aufsichtsbehörde zu genehmigen. ${ }^{12}$

\footnotetext{
6 Ibid.

7 STOCKER (Fn. 4), S. 71f.

8 Art.17 FZG. Der Mindestbetrag setzt sich zusammen aus den eingebrachten Eintrittsleistungen samt Zinsen sowie auf den von der versicherten Person während der Beitragsdauer geleisteten Beiträge samt einem Zuschlag von 4 Prozent pro Altersjahr ab dem 20. Al tersjahr, höchstens aber von 100 Prozent.

9 Art.18a Abs.1FZG; Art. 279 Abs.1 BVV 2.

10 Art. 27h Abs.1 BVV 2; Die jeweilige VE kann jedoch auf freiwilliger Basis bei einem individuellen Austritt im Rahmen einer TL die ausscheidenden Destinatäre an den Rückstellungen und Wertschwankungsreserven Anteil haben lassen (WILSON [Fn. 2], Rz.207 f.).

11 Zum Ganzen vgl. WILSON (Fn. 2), Rz.73ff.

12 Art. 53b Abs. 2 und 61 ff. BVG; WILSON (Fn. 2), Rz. 392.
} 
7 Die Voraussetzungen einer TL sind vermutungsweise erfüllt, wenn eine der drei folgenden Tatbestände erfüllt ist: 13

a) es erfolgt eine erhebliche Verminderung der Belegschaft;

b) eine Unternehmung wird restrukturiert; oder

c) der Anschlussvertrag wird aufgelöst.

Grundsätzlich reicht eine der drei Voraussetzungen, um die TL auszulösen. Im Rahmen der einzelnen Voraussetzungen lässt das Bundesgericht aus Praktikabilitätsgründen jedoch zusätzliche Kriterien zu, was im Folgenden noch gezeigt wird. ${ }^{14}$

9 Bei der Definition der Voraussetzungen und Folgen der TL im TL-Reglement hat die VE einen relativ grossen $\mathrm{Er}$ messensspielraum. ${ }^{15}$ Das Bundesgericht äusserte sich in der Vergangenheit mehrmals zur Rechtmässigkeit und Gesetzeskonformität von TL-Reglementen und konkretisierte die drei genannten Tatbestände.

\section{Erhebliche Verminderung der Belegschaft}

Das Gesetz regelt nicht explizit, wann die Erheblichkeit gegeben ist. Obwohl man immer den Einzelfall in Betracht ziehen muss, kann grundsätzlich von einer erheblichen Verminderung ausgegangen werden, wenn mindestens 10\% des Personalbestandes das Unternehmen verlassen. ${ }^{16}$ Dieser Prozentsatz ist nicht als fixe Grösse zu verstehen und kann nicht generell auf alle VE angewendet werden. ${ }^{17}$ Eine TL kann deshalb auch unter oder oberhalb dieser Schwelle vorliegen, vor allem bei sehr grossen oder besonders kleinen Unternehmen. ${ }^{18}$ Hingegen wurde eine generelle reglementarisch festgelegte Grösse von 15\% vom Bundesgericht als zu hoch erachtet. ${ }^{19}$ Praxisgemäss ist in den TL-Reglementen entweder von absoluten (z.B. 25 Personen) oder relativen (z.B. 10\% der Belegschaft) Zahlen die Rede, wobei auch eine Kombination möglich ist (z.B. 20\% der Belegschaft, mindestens

13 Art. $53 b$ Abs.1 BVG; zur Zulässigkeit von weiteren Kriterien vgl. WILSON (Fn. 2), Rz.159ff.

14 BGE136V322 E.10.2.

15 Kritisch demgegenüber LAURENCE UTTINGER, Zukünftige Lösungen für Teilliquidationen: Schnellere Verfahren, Schweizer Personalvorsorge 8/2019, S. 6of.; vgl. auch WILSON (Fn. 2), Rz.73 ff.; BENNO AMBROSINI / ANDREA TRÜSSEL, Teilliquidation - Teil1: Welche Schwellenwerte sind zulässig und gerecht?, Schweizer Personalvorsorge $2 / 2018$, S. 88.

16 Urteil des Bundesgerichts 2A.48/2003 vom 26.Juni 2003 E.3.1; WILSON (Fn. 2), Rz.114; STOCKER (Fn. 4), s. 95; kritisch dazu AMBROSINI/ TRÜSSEL, Teilliquidation - Teil1 (Fn.15), S. 88.

17 BGE136V 322 E.8.3.

18 STOCKER (Fn.4), S. 96.

19 BGE136V 322 E. 9. jedoch 100 Personen bei einer Anschlussvereinbarung bis zu 1000 Personen). ${ }^{20}$

Freiwillige Austritte und solche, die im gegenseitigen 11 Einvernehmen erfolgen, sind für die TL grundsätzlich unbeachtlich. ${ }^{21}$ Die Regeln über die TL bei VE greifen nur, wenn die Personalreduktion vom Arbeitgeber ausgeht. Anders sieht es aus, wenn ein «schleichender» Personalabbau erfolgt. Sind bspw. zwei zeitlich nahestehende Entlassungswellen auf dasselbe wirtschaftliche Ereignis $^{22}$ zurückzuführen, sind sämtliche Destinatäre in die TL einzubeziehen. ${ }^{23}$ Gewöhnlich wird hier auf einen Zeitraum von bis zu fünf Jahren abgestellt. ${ }^{24}$

\section{Restrukturierung}

Bei einer Restrukturierung müssen zwei Bedingungen 12 erfüllt sein. ${ }^{25}$ Erstens muss das betroffene Unternehmen neu- oder umgestaltet werden. Darunter fallen etwa die Schliessung eines Betriebsteils, die Zusammenlegung von Abteilungen, der Verkauf einer Tochterfirma ${ }^{26}$ aber auch das Outsourcing von internen Dienstleistungen. ${ }^{27}$ Die Restrukturierung muss zudem eine gewisse Schwere aufweisen; eine blosse Änderung der Führungsstruktur oder eine Sitzverlegung allein erfüllen den Tatbestand nicht. ${ }^{28}$

Zweitens muss die Umgestaltung mit einer Änderung des 13 Personalbestands einhergehen, welche nicht nur in einer Verminderung, sondern auch in einer Erhöhung bestehen kann. ${ }^{29}$ Besonders deutlich wird dies bei einer Fusion, wo zwar eine Änderung im Personalbestand stattfindet, nach der Restrukturierung jedoch mehr Personen für das jeweilige Unternehmen tätig sein können. ${ }^{30}$ Die Erheblichkeit gemäss Art. $53 b$ Abs. 1 lit. a BVG ist also bei

20 WILSON (Fn. 2), Rz.116f.; vgl. a uch das Merkblatt der BVG- und StiftungsaufsichtZürich, S. 2; vgl. ferner das Teilliquidationsreglement der Post-Pensionskasse, Art. 2 Abs. 3;

21 ISABELLE VETTER-SCHREIBER, in: Vetter-Schreiber (Hrsg.), BVG/ FZG Kommentar, Orell Füssli Kommentar, 3. Aufl., Zürich 2013, Art. 53-53b, N11 ff.

22 WILSON (Fn. 2), Rz.125 ff.; LUCREZIA GLANZMANN-TARNUTZER, Bekanntes und Neues zur Teilliquidation von Vorsorgeeinrichtungen, AJP 2019, S. 598.

23 Urteil des Bundesgerichts 2A.48/2003 vom 26.Juni 2003 E.3.1.

24 BGE128II394 E. 6.4 .

25 Zum Ganzen vgl. BENNO AMBROSINI / ANDREA TRÜSSEL, Teilliquidation - Teil 2: Wann sollten Teilliquidationen durchgeführt werden?, Schweizer Personalvorsorge 4/2018, S.101.

26 UELI KIESER, in: Schneider/Geiser/Gächter (Hrsg.), BVG und FZG, Stämpflis Handkommentar, Bern 2010, Art. $53 b$ N18.

27 BGE136 V 322 E. 8.3; STOCKER (Fn. 4), s.107f.

28 WILSON (Fn. 2), Rz.133.

29 Botschaft vom 1. März 2000 zur Revision des Bundesgesetzes über die berufliche Alters-, Hinterlassenen- und Invalidenvorsorge (BVG) (BBl 2000 2637), S. 2696; vgl. auch WILsON (Fn. 2), Rz.134ff.

30 AMbrosini/TRÜSSEL, Teilliquidation - Teil2 (Fn. 25), S. 101. 
der Restrukturierung nicht Voraussetzung und es kann bereits eine Veränderung des Personalbestands von 1-5\% ausreichen. ${ }^{31}$ Diese Zahlen sind nicht als absolut fixe Zahlen zu verstehen. Vielmehr ist der Grundsatz der Verhältnismässigkeit zu beachten, gerade wenn die Restrukturierung nur sehr wenig Vorsorgekapital betrifft oder der Deckungsgrad der VE praktisch unverändert bleibt. ${ }^{32}$

\section{Auflösung eines Anschlussvertrages}

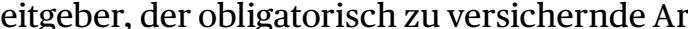
beitnehmer beschäftigt, muss entweder eine VE errichten oder - was in der Praxis meistens der Fall ist - einer solchen mittels Anschlussvertrag beitreten. ${ }^{33}$ Für den Anschlussvertrag wird oft eine Mindestdauer vereinbart mit einer anschliessenden Kündigungsfrist von sechs Monaten. der Auflösung eines Anschlussvertrages häufig auf ein zusätzliches Kriterium wie bspw. das Betroffensein einer Mindestanzahl von Destinatären abgestellt. ${ }^{37}$ Wären solche Zusatzkriterien nicht erlaubt, würde sich die VE in einem Zustand permanenter TL befinden, was gerade bei Anschlussverträgen mit einer sehr kleinen Zahl von Destinatären einen unverhältnismässigen Aufwand zur Folge hätte. ${ }^{38}$ Deshalb sehen die TL-Reglemente meist vor, dass mindestens 7-10\% des Gesamtbestandes der Destinatäre und/oder 7-10\% der Deckungskapitalien betroffen sein müssen. ${ }^{39}$

31 BGE136V 322 E. 10.4; Zum Ganzen vgl. STOCKER (Fn.4), S.100ff.; WILSON (Fn. 2), Rz.138ff.

32 STOCKER (Fn. 4), S. 102.

33 Art.11 BVG.

34 Art.11 Abs.3bis BVG; vgl. auch Urteil des Bundesgerichts 9C_409/2019 vom 5. Mai 2020; zur fehlenden Mitwirkung des Personals vgl. OLIVER M. PETER, «Abwicklungsstörungen» bei Wechsel des Berufsvorsorgeträgers, Schweizerische Zeitschrift für Sozialversicherung und berufliche Vorsorge 2018, S. 89.

35 Urteil des Bundesgerichts 9C_409/2019 vom 5. Mai 2020 E.4.3.1

36 Art. 11 Abs. $3^{\text {ter }}$ BVG.

37 YOLANDA MÜLLER, Teilliquidation und Teilliquidationsreglement: Auflösung eines Anschlussvertrags, Schweizer Personalvorsorge 8/2017, S. 92 f.

38 BGE143V 200 E. 4.2.2; WILSON (Fn. 2), Rz.151ff.; GLANZMANN-TARNUTZER (Fn. 22), S. 598

39 STOCKER (Fn. 4), s.111.
Das Bundesgericht teilt grundsätzlich diese Meinung aus 17 Gründen der Verhältnismässigkeit. ${ }^{40}$ Eine Reglementsbestimmung, die besagte, dass die TL nicht schon bei Auflösung eines einzelnen Anschlussvertrages durchzuführen ist, sondern erst, wenn die Anschlussverträge von mindestens 10\% der angeschlossenen Betriebe aufgelöst werden, erachtete es aufgrund der Verletzung des Gleichheitsgebotes als ungültig. ${ }^{41}$ Konsequent durchgedacht würde nämlich die Auflösung eines Anschlussvertrages eines Unternehmens mit einer Vielzahl von Angestellten (aber weniger als 10\% der Destinatäre) keine TL nach sich ziehen, die Auflösung von Anschlussverträgen von Kleinstunternehmen mit viel weniger Arbeitnehmenden jedoch schon.

In einem weiteren Fall sah ein TL-Reglement vor, dass 18 die Voraussetzung für eine TL bei Auflösung eines Anschlussvertrages erfüllt ist, sofern mindestens $2 \%$ der Versicherten aus der VE ausscheiden. ${ }^{42}$ Das Bundesgericht erachtete diese Bestimmung als rechtmässig, präzisierte jedoch, dass auch Vertragsauflösungen unterhalb der 2\%-Marke zu berücksichtigen seien. Demnach sind auch Kleinstanschlüsse zu beachten, wenn die Anschlussverträge wegen des gleichen wirtschaftlichen Ereignisses, das zur TL führte, aufgelöst würden. ${ }^{43}$

\section{Bemessung des Anspruchs}

Sind die Voraussetzungen einer TL gegeben, ist als 19 Nächstes der Umfang des Anspruchs der Destinatäre zu berechnen. Die TL muss unter Berücksichtigung des Gleichbehandlungsgrundsatzes und nach fachlich anerkannten Grundsätzen durchgeführt werden. ${ }^{44}$ Welcher Anspruch der jeweilige Destinatär im Rahmen einer TL hat, bemisst sich nebst der Voraussetzung, ob überhaupt eine TL vorliegt, danach, ob ein individueller bzw. kollektiver Austritt erfolgt und ob die jeweilige VE über- oder unterkapitalisiert ist. Der Einfachheit halber wird im Folgenden davon ausgegangen, dass es sich um einen kollektiven Austritt handelt, bei dem nebst der Austrittsleistung und den freien Mitteln auch Rückstellungen und Wertschwankungsreserven berücksichtigt werden müssen. ${ }^{45}$ Bei einer Unterdeckung ist zudem der Fehlbetrag näher zu betrachten.

\section{WILSON (Fn. 2), Rz.155.}

41 BGE143 V 200 E. 4.3; AMBRosINI/TRÜSSEL, Teilliquidation - Teil 1 (Fn.15), S.87f.

42 BGE145V22 E. 4.1ff.

43 BGE145V 22 E. 4.2.

44 Art. 53d Abs.1BVG.

45 Vgl. zudem das Praxisbeispiel bei BENNO AMBROSINI / JÜRG WALTER, Teilliquidationen: Fragestellungen aus der Praxis, Schweizer Personalvorsorge 8/2019, S. $39 \mathrm{ff}$. 


\section{Freie Mittel}

20 Vereinfacht gesagt fällt darunter dasjenige Vermögen, welches nicht zur Deckung der gesetzlichen und reglementarischen Verpflichtungen gehört. ${ }^{46}$ Gemäss Swiss GAAP FER $26^{47}$ können freie Mittel erst entstehen, wenn die notwendigen Rückstellungen und Wertschwankungsreserven gebildet sind. ${ }^{48}$ In der Praxis entstehen die freien Mittel etwa durch Mutationsgewinne, d.h. frei werdende Deckungskapitalien beim Austritt eines Destinatärs. ${ }^{49}$ Zudem spielen freiwillige Zuwendungen des Arbeitgebers und vor allem Kapitalerträge eine wichtige Rolle. ${ }^{50}$

\section{Rückstellungen}

21 Zusätzlich zu den individuellen Deckungskapitalien werden technische Rückstellungen als Sicherheitsmassnahme für Leistungsversprechen gebildet, die durch die Beiträge der Destinatäre nicht genügend gedeckt sind oder Risikoschwankungen unterliegen.51 Deren grundsätzliche Bemessung muss im TL-Reglement bezeichnet werden, die genaue Bemessung erfolgt in einem separaten Rückstellungsreglement. ${ }^{52}$ Das Bundesgericht hat jedoch jüngst entschieden, dass wenn sich im Rahmen einer TL die Risikofähigkeit einer VE schlagartig und grundlegend ändert, neue Rückstellungen auch ohne reglementarische Grundlage gebildet werden können. ${ }^{53}$

22 Die anteilsmässige Übertragung erfolgt wie bereits erwähnt nur im Falle eines kollektiven Austritts und zwar auch dann, wenn die neue VE keine gleichartige Bildung von Rückstellungen vorsieht. ${ }^{54}$ Entscheidend ist also immer der Umstand bei der abgebenden VE. ${ }^{55}$ Die Schweizerische Kammer für Pensionskassen-Experten empfiehlt die Bildung von Rückstellungen für folgende Fälle56:

\footnotetext{
46 STOCKER (Fn. 4), S.126; WILSON (Fn. 2), Rz.183ff.

47 Fachempfehlungen zur Rechnungslegung (Generally Accepted Ac counting Principles) der Stiftung für Fachempfehlungen zur Rechnungslegung (FER). Standard 26 umfasst die Rechnungslegung von Vorsorgeeinrichtungen (SWISS GAAP FER 26).

48 Swiss GAAPFER 26, Empfehlung 2.

49 WILSON (Fn. 2), Rz.184.

50 STOCKER (Fn. 4), s.128.

51 BGE144 V120 E. 2.1 mit Verweis auf WILSON (Fn. 2), Rz. 211.

52 Ar t. $65 b$ BVG i.V.m. Art. $48 e$ BVV 2; zum Ganzen vgl. ERICH PETER, Die Verteilung von Rückstellungen bei Teilliquidationen - das korrekte Vorgehen, Schweizerische Zeitschrift für Sozialversicherung und berufliche Vorsorge (SZS) 2014, S. $83 \mathrm{ff}$.

53 BGE144 V 264 E.3.4.1; GLANZMANN-TARNUTZER(Fn. 22), s. 603.

54 Art.27h BVV 2; s. auch Bundesamt für Sozialversicherungen, Mittei lung Nr.117 vom 31. März 2010über die berufliche Vorsorge, Rz. 736.

55 Vgl. auch Urteil des Bundesgerichts 9C_615/2017 vom 16. März 2018 E.1.2.3.

56 Fachrichtlinie FRP 2 der Schweizerischen Kammer der Pensionskassen-Experten; GLANZMANN-TARNUTZER (Fn. 22), S. 602.
}

- Zunahme der Lebenserwartung;

- Tod und Invalidität;

- Schwankungen im Risikoverlauf bei Rentnerbeständen;

- Pensionierungsverluste;

- Pendente und latente Leistungsausfälle;

- Senkung des technischen Zinssatzes; oder

- Rentenerhöhungen.

\section{Wertschwankungsreserven}

Diese Reserven dienen der Absicherung von Wert- 23 schwankungsrisiken von Vermögensanlagen. ${ }^{57}$ Gleich wie Rückstellungen werden auch sie nur bei einem kollektiven Austritt anteilsmässig ausbezahlt, unabhängig davon, ob die neue VE eine gleichartige Bildung von Rückstellungen vorsieht. 58

\section{Fehlbeträge bei Unterdeckung}

Die Überwälzung eines Fehlbetrages kommt nur bei ei- 24 ner Unterdeckung infrage. Eine solche liegt vor, wenn am Bilanzstichtag notwendiges Vorsorgekapital nicht durch das verfügbare Vorsorgevermögen gedeckt ist. ${ }^{59}$ In diesem Fall muss ein Fehlbetrag aufgrund des Gleichbehandlungsgebotes abgezogen werden, weil sonst die verbleibenden Destinatäre benachteiligt würden. ${ }^{60} \mathrm{Um} \mathrm{zu}$ verhindern, dass Rückstellungen und Wertschwankungsreserven nur gebildet werden, um die freien Mittel zu schmälern, ist bei TL-Reglementen deshalb zu beachten, wie diese Fehlbeträge berechnet werden. ${ }^{61}$ Der anteilmässige Abzug von Fehlbeträgen ist jedoch nur zulässig, sofern das obligatorische Altersguthaben (BVGMinimalleistung) nicht geschmälert wird, d.h. jene Beiträge, die der Destinatär einbezahlt hat.62 Auch im Rahmen einer TL darf dieses Guthaben demnach nicht angetastet werden und der ausscheidende Destinatär hat Anspruch auf die volle, ungekürzte Austrittsleistung. ${ }^{63}$

\section{Rechtsweg}

Grundsätzlich wird eine TL von der VE autonom durch- 25 geführt. Die Destinatäre werden über die TL informiert und sie erhalten Einsicht in den Verteilungsplan. ${ }^{64}$

\footnotetext{
57 WILSON (Fn. 2), Rz.216ff.; zur Verteilung von Wertschwankungsreserven in bar vgl. BGE146 V 28 E. 4.4.1; BGE131 II 525 E. 6.

58 Art.27h Abs.1BVV2.

59 Art. 44 Abs.1BVV 2.

60 WILSON (Fn. 2), Rz.227.

61 Art. 53d Abs. 4 BVG; STOCKER (Fn. 4), S. 127.

62 Art.53d Abs.3 i.V.m. Art.15 BVG.

63 BGE138V303 E.3.1.

64 Art. 53d Abs. 5 BVG; Wilson (Fn. 2), Rz. 351 ff.
} 

setzungen der TL einer VE erheblichen Spielraum. In der Praxis ist deshalb primär der Fall interessant, in dem eine TL aufgrund der gegebenen Voraussetzungen durchzuführen wäre, die VE jedoch darauf verzichtet. Aus Sicht eines ausscheidenden Destinatärs ist dies dann nachteilig, wenn er keine Anteile an den freien Mittel und allenfalls (Wertschwankungs-)reserven erhält. ${ }^{65}$ Falls die VE nicht von sich aus tätig wird, können nebst den Destinatären ${ }^{66}$ und Rentnern auch die übernehmende VE oder der neue Arbeitgeber, der sich für die ausscheidenden Destinatäre einsetzt, dies verlangen. ${ }^{67}$ Wenn die VE auch nach dieser Aufforderung untätig bleibt, kann dies bei der kantonalen Aufsichtsbehörde angezeigt werden. ${ }^{68}$ Diese kann auch von Amtes wegen tätig werden, wenn sie bei der Prüfung der Bilanz und Betriebsrechnung der VE bemerken sollte, dass die Voraussetzungen einer TL vorliegen würden. ${ }^{69}$

27 Gemäss Art. 53d Abs. 6 BVG besteht das Recht, die Voraussetzungen, das Verfahren und den Verteilungsplan bei der zuständigen kantonalen Aufsichtsbehörde «überprüfen und entscheiden zu lassen». Obwohl diese Formulierung eine generelle Überprüfung ohne spezifische Gründe zulassen würde, empfiehlt es sich, möglichst genau zu zeigen, inwiefern Recht verletzt wurde. ${ }^{70}$

Das konkrete Verfahren richtet sich in erster Linie nach dem jeweiligen TL-Reglement. Diese sehen üblicherweise ein internes Verfahren vor, bei der innerhalb von 30 Tagen seit Bekanntgabe Einsprache beim obersten Leitungsorgan (z.B. dem Stiftungsrat) erhoben werden muss. ${ }^{71}$ Gemäss bundesgerichtlicher Rechtsprechung sind VE berechtigt, solche internen Fristen festzulegen. ${ }^{72}$ Der Einspracheentscheid kann an die kantonale Aufsichtsbehörde und im Anschluss ans Bundesverwaltungs-73 und schliesslich ans Bundesgericht ${ }^{74}$ weitergezogen werden. Je nach TL-Reglement führt der Rechtsweg also über vier Instanzen. Die TL darf erst vollzogen werden, wenn ein rechtskräftiger Entscheid vorliegt.

\footnotetext{
65 Zum Ganzen vgl. WILSON (Fn. 2), Rz.415ff.

66 Zur fehlenden Aktivlegitimation einer Witwe vgl. BGE145 V343 E. 2.

67 Art. 53d Abs. 6 Satz1 BVG; BGE140 V 22 E. 4.2; Urteil des Bundesgerichts 2A.14/2006 vom 4. Mai 2006 E. 2.1.; GLANZMANN-TARNUTZER (Fn. 22), S. 599; WILSON (Fn.2), Rz.473 ff.

68 Art. 53d Abs. 6 BVG.

69 WILSON (Fn. 2), Rz.416f.

70 WILSON (Fn.2), Rz. $479 \mathrm{ff}$.

71 WILSON (Fn. 2), Rz.377ff.; vgl. auch die Muster-Reglemente bei WILSON (Fn. 2), S.169ff., und STOCKER (Fn. 4), S. $193 \mathrm{ff}$.

72 Urteil des Bundesgerichts 9C_15/2019 vom 21. Mai 2019 E.3.1.1f.

73 Art. 53d Abs.6BVG.

74 Art. 82 ff. BVG; Beschwerde in öffentlich-rechtlichen Angelegenheiten.
}

\section{Fazit}

Die vorstehende Übersicht zeigt, dass bei einer M\&A 29 Transaktion und bei Restrukturierungen in Bezug auf die VE genau zu prüfen ist, ob die gesetzlichen Voraussetzungen einer TL vorliegen und wenn ja, wie sich die Ansprüche der Destinatäre bemessen. Die folgenden Punkte sollten dabei beachtet werden:

Die wichtigsten gesetzlichen Grundlagen finden sich im 30 FZG, dem BVG und der BVV 2. Danach liegt eine TL einer VE vor, wenn eine der drei folgenden Voraussetzungen erfüllt sind:

1) Eine erhebliche Verminderung der Belegschaft liegt vor, wenn der Personalbestand aus wirtschaftlichen Gründen um mindestens 10\% vermindert wird, wobei der Einzelfall analysiert werden muss. Freiwillige Austritte sind grundsätzlich unbeachtlich.

2) Eine Restrukturierung wird angenommen, wenn sich der Personalbestand um 1-5\% verändert und das Unternehmen dabei neu- oder umgestaltet wird.

3) Die Auflösung des Auflösungsvertrages kann an Bedingungen geknüpft werden, z.B. daran, dass eine Mindestanzahl an Destinatären oder ein bestimmter Teil des Deckungskapitals betroffen sein muss.

Die TL-Reglemente der VE konkretisieren die gesetzli- 31 chen Regelungen. Die Praxis zeigt jedoch, dass TL von VE eher zögerlich durchgeführt werden und dass die Aufsichtsbehörden nicht angerufen werden und sie die TL-Reglemente zu wenig kritisch prüfen. Gerade von Seiten der austretenden Destinatäre und des neuen Arbeitgebers sind deshalb die Voraussetzungen und die daraus resultierenden Ansprüche genau zu prüfen.

Die nachstehende Tabelle ${ }^{75}$ bietet einen Überblick der 32 verschiedenen Ansprüche.

\begin{tabular}{|l|c|c|c|c|c|c|}
\cline { 2 - 7 } \multicolumn{1}{c|}{} & \multicolumn{4}{l}{ Ansprüche } \\
\cline { 2 - 7 } \multicolumn{1}{c|}{} & freie Mittel & \multicolumn{2}{l|}{$\begin{array}{l}\text { Rückstellun- } \\
\text { gen und Wert- } \\
\text { schwankungs- } \\
\text { reserven }\end{array}$} & \multicolumn{2}{l}{$\begin{array}{l}\text { Fehlbetrag } \\
\text { (Unterdeckung) }\end{array}$} \\
\cline { 2 - 7 } \multicolumn{1}{c|}{} & indiv. & koll. & indiv. & koll. & indiv. & koll. \\
\hline $\begin{array}{l}\text { Freizügigkeits- } \\
\text { fall }\end{array}$ & $\times$ & $\times$ & $\times$ & $\times$ & $\times$ & $\times$ \\
\hline $\begin{array}{l}\text { individueller } \\
\text { Austritt bei TL }\end{array}$ & $\checkmark$ & $\times$ & $\times$ & $\times$ & $\checkmark$ & $\times$ \\
\hline $\begin{array}{l}\text { kollektiver } \\
\text { Austritt bei TL }\end{array}$ & $\checkmark$ & $\checkmark$ & $\times$ & $\checkmark$ & $\checkmark$ & $\times$ \\
\hline
\end{tabular}

75 Angelehnt an STOCKER (Fn. 4), s. 163. 